\title{
TRANSMISSION OF ULTRASONIC CONTRAST THROUGH THE LUNGS
}

\author{
Richard S. Meltzer,* Otto E. H. Sartorius, Charles T. Lancée, Patrick W. \\ Serruys, Pieter D. Verdouw, Catharina E. Essed and Jos Roelandt \\ Thoraxcenter, Erasmus University and Dijkzigt Hospital, Rotterdam, The Netherlands
}

(First received 15 August 1980; and in final form 27 January 1981)

\begin{abstract}
The pulmonary capillary bed normally removes echocardiographic contrast from the circulation, so contrast injected peripherally or on the right side of the heart is not seen on the left side of the heart in the absence of intracardiac or intrapulmonary shunts. Based on recent advances in the theoretic understanding of microbubble physiology, we propose several theoretic methods for causing the transmission of ultrasonic contrast through the lungs to enable opacification of the left side of the heart. Three of these methods are tested: (1) injection of ether, an organic compound which may pass the pulmonary capillaries in the liquid phase and cavitate in the pulmonary veins to yield left heart echo contrast, (2) injection of hydrogen peroxide, a substance which chemically decomposes on the left side of the heart to yield gaseous oxygen that can be imaged as echo contrast, and (3) injections of $5 \%$ dextrose in the pulmonary wedge position. The first two methods were tested in anesthetized pigs, and the third method in humans and anesthetized rabbits. All methods could cause transmission of echocardiographic contrast through the lungs. There were no adverse reactions in the human subjects. Pulmonary wedge injections in rabbits were associated with one large and three small myocardial infarctions out of 7 animals sacrificed $24 \mathrm{hr}$ later. We conclude that transmission of echocardiographic contrast through a capillary bed is feasible though potentially dangerous.
\end{abstract}

Key words: Echocardiography, Ultrasound contrast, Ether, Hydrogen peroxide.

\section{INTRODUCTION}

Echocardiographic contrast was first reported during injections of indocyanine green in left sided heart cavities (Gramiak and Shah, 1968). Since then numerous studies have commented on the use of echocardiographic contrast on the left side of the heart, but in the absence of right to left shunts all have necessitated cardiac catheterization and direct intracardiac injection to create left heart echocardiographic contrast (LHEC) (Gramiak et al., 1969; Feigenbaum et al., 1970; Sahn et al., 1974; Kerber et al., 1974).

Noninvasive or minimally invasive creation of LHEC would be helpful in left sided structure identification, left to right shunt detection, and perhaps in the detection of mitral and/or aortic regurgitation and proximal coronary artery narrowing. It might also have use in noncardiac arterial ultrasonography.

Lack of theoretic understanding of the nature of ultrasound contrast and the physiology of its removal by the lung has precluded efforts to create LHEC without per-

*Clinician-Scientist Awardee of the American Heart Association. forming left heart catheterization. Our recent experimental (Meltzer et al., 1980a; Meltzer et al., 1980b) and clinical (Meltzer et al., 1980 c) work in this field has enabled us to form several concepts about methods that might cause contrast to pass the pulmonary capillary bed and yield LHEC. This communication reports on animal experiments during which three of these methods were tested, comments on our clinical experience with pulmonary wedge injections and discusses the relevance of these findings in relation to the ultimate goal of noninvasive LHEC creation.

\section{THEORETIC BACKGROUND}

Recent studies suggest that microbubbles of gas injected with liquid solutions are the source of ultrasonic contrast (Meltzer et al., 1980b; Barrera et al., 1978). The high contrast is due to the extremely large difference in acoustic impedance between gas and liquids, much larger than occuring between various non-air containing biologic tissues. In the absence of pulmonary arteriovenous shunting, the lungs like any capillary bed remove ultrasonic contrast. This is because gas bubbles considerably larger than the pulmonary 
capillary diameter of approx. $8 \mu$ are stopped by the "sieve" action of the capillaries. On the other hand, due to surface tension, bubbles small enough to pass the capillary bed have an internal pressure significantly higher than the ambient pressure in the pulmonary veins. Gas inside the microbubbles therefore dissolves down its concentration gradient into the surrounding fluid. Consequently the bubble becomes smaller, internal pressure larger, and the process continues in an accelerating manner until the microbubble of gas is totally absorbed. We calculate that the duration of this process is shorter than the pulmonary capillary to the left heart circulation time, and this is the reason that the lungs remove peripheral injected ultrasonic contrast (Meltzer et al., 1980a).

"Spontaneous" ultrasonic contrast has been noted in patients with mitral valve prostheses (Schuchman et al., 1975; Preis et al., 1980). Though this has been attributed to fibrin or particulate matter (Schuchman et al., 1975) or to oxygen released due to hemolysis (Preis et al., 1980), we believe that it occurs mainly in patients with local areas of pressure below the combined vapor pressure of blood and partial pressures of all dissolved gasses, due to the Bernouilli principle in jets of regurgitant blood in the low pressure left atrium. The foreign body provided by an artificial valve may act as a source of nuclei to allow cavitation to take place with a lesser degree of supersaturation than needed with an intact endocardium.

These considerations allow proposal of the following mechanisms for the creation of LHEC:

(1) A liquid could be administered intravenously which would pass through the lungs in liquid state and boil on the left side of the heart.

(2) A liquid or combination of substances could be administered intravenously which would pass the lungs and undergo a chemical reaction on the left side of the heart, yielding a gas.

(3) Bubbles of gas might be forced through the capillary bed by the increased pressure that could be applied locally by an injection through a catheter in the pulmonary wedge position.

(4) A "super" surfactant might stabilize microbubbles small enough to pass the pulmonary capillaries until they reach the left heart.
(5) A more solid coat might protect a "mini-microbubble" from dissolving but still be small enough to allow transcapillary transmission (Bommer et al., 1980).

(6) A gas might be inhaled which, due to its composition and airway pressure, could sufficiently alter the partial pressures of gases in the pulmonary capillaries so intravenously injected microbubbles would grow instead of decay.

(7) High energy ultrasound could be focussed in the left atrium or ventricle until cavitation occurs.

(8) The concept of "rectified diffusion" might be used to enable lower, nontoxic energies of ultrasound to cause growth rather than decay of small microbubbles which can pass the pulmonary capillary bed (Higashiizumi et al., 1979; Plessett and Prosperetti, 1977).

(9) An ultrasonic contrast medium in the liquid phase might be developed, allowing transcapillary transmission as a liquid, without the difficulties faced by gas bubbles.

(10) A combination of any of the above methods.

In the following set of experiments we tested the efficacy of the first three methods. Since we were already aware of the occasional efficacy of wedge injections in causing LHEC (Meltzer et al., 1980c; Bommer et al., 1978; Reale et al., 1980), we decided to examine potential toxicity of this technique.

\section{METHODS}

\section{Method 1: physical cavitation-ether}

Ten pigs were anesthetized with barbituurates and placed on a respirator. Surface ECG and intra-arterial pressure were monitored continuously. In each pig the thorax was opened by a median sternotomy and a Krautkramer-Branson $3.5 \mathrm{MHz}$ ultrasonic transducer was sutured directly onto the left ventricular epicardium and used to search for LHEC. Rapid hand injections were made in the right heart or proximal pulmonary artery via Swan-Ganz balloon catheter. Two control injections of $5-8 \mathrm{~cm}^{3}$ normal saline were monitored by $M$-mode tracings to insure that no resting intracardiac or pulmonary arteriovenous shunts existed. Each pig had one or more of the following amounts of diethyl ether- $0.5,1,1.5,2$ or $3 \mathrm{~cm}^{3}$-followed by a $3-\mathrm{cm}^{3}$ "flush" of normal saline (Table 1). Following each injection the left ventricular 
Table 1. Ether injections

\begin{tabular}{|c|c|c|c|}
\hline Pig no. & $\begin{array}{l}\text { Injected } \\
\text { volume (cc) }\end{array}$ & $\begin{array}{l}\text { Contrast with } \\
5 \mathrm{cc} \text { saline }\end{array}$ & $\begin{array}{l}\text { Contrast with } \\
\text { ether }\end{array}$ \\
\hline \multirow[t]{2}{*}{1} & 0,5 & - & - \\
\hline & 1,0 & & + \\
\hline 2 & 1,0 & + & \\
\hline \multirow[t]{2}{*}{3} & 0,5 & - & - \\
\hline & 1,0 & & - \\
\hline \multirow[t]{3}{*}{4} & 0,5 & - & - \\
\hline & 1,0 & & - \\
\hline & 1,5 & & + \\
\hline 5 & 1,0 & - & + \\
\hline 6 & 2,0 & - & + \\
\hline \multirow[t]{2}{*}{7} & 1.5 & - & + \\
\hline & 2,0 & & + \\
\hline \multirow[t]{2}{*}{8} & 1,0 & - & - \\
\hline & 2,0 & & - \\
\hline 9 & 1,0 & - & + \\
\hline 10 & 1,0 & + & \\
\hline
\end{tabular}

+ left heart contrast obtained

-: no left heart contrast seen

echocardiogram, ECG and arterial pressure were recorded for $30-60 \mathrm{sec}$ and monitored visually on the oscilloscope for several minutes further. Subsequent injections were not made until ECG and pressure returned to baseline.

Method 2: chemical reaction-hydrogen peroxide

Twelve pigs were anesthetized with barbiturates and placed on a respirator, with an ultrasonic transducer sutured to the left ventridular epicardium as in method 1 . Control injections of normal saline were monitored by $M$-mode tracings to insure that no cardiac or pulmonary shunting existed. Right heart injections of $0.3-5 \mathrm{~cm}^{3}$ of hydrogen peroxide followed by a $3-\mathrm{cm}^{3}$ normal saline "flush" were then performed. The volumes and concentrations employed in each pig are listed in Table 2. Sometimes more than 1 injection was performed with the same volume and concentration.

Method 3 (animal studies): pulmonary wedge injections

Seven adult rabbits were anesthetized with fluanison $0.5 \mathrm{mg} / \mathrm{kg}$. Number 6 French SwanGanz catheters were advanced to the wedge position under fluoroscopic and pressure control. They were inflated with $0.5-0.75 \mathrm{~cm}^{3}$ air and 3-10 repeated injections of $3-5 \mathrm{~cm}^{3}$ normal saline were performed. In most injections the small amount of air normally trapped in the 3-way stopcock was not assiduously excluded as is our practice in clinical injections. The animals were sacrificed 1 day later. The lungs, heart and kidneys were examined grossly and by light microscopy.

Method 3 (human studies): pulmonary wedge injections

During routine cardiac catheterization in patients, pulmonary wedge injections were performed either through a 7 French Cournand $(n=9)$, a 7 French Swan-Ganz catheter in the wedge position but without an inflated balloon $(n=3)$, or both catheters $(n=5)$. LHEC was monitored by two-dimensional echocardiography. Ten patients had X-ray angiograms performed during a hand injection of a new nonionic radiographic contrast agent-Amipaque ${ }^{\circledR}$, Nyegaard \& Co., Oslo, Norway (Enge et al., 1977) in the same 
Table 2. $\mathrm{H}_{2} \mathrm{O}_{2}$ injections

\begin{tabular}{|c|c|c|c|c|}
\hline Pig no. & $\begin{array}{l}\mathrm{H}_{2} \mathrm{O}_{2} \text { concen- } \\
\text { tration }\end{array}$ & $\begin{array}{l}\mathrm{H}_{2} \mathrm{O}_{2} \\
\text { volume (cc) }\end{array}$ & $\begin{array}{l}\text { Contrast } \\
\text { with saline }\end{array}$ & $\begin{array}{l}\text { Contrast } \\
\text { with } \mathrm{H}_{2} \mathrm{O}_{2}\end{array}$ \\
\hline \multirow[t]{3}{*}{1} & $30 \%$ & 0,2 & - & + \\
\hline & & 0,3 & & + \\
\hline & & 0.5 & & + \\
\hline \multirow[t]{2}{*}{2} & 308 & 0.2 & - & + \\
\hline & & 0,3 & & + \\
\hline \multirow[t]{2}{*}{3} & 308 & 0,2 & - & - \\
\hline & & 0.3 & & - \\
\hline 4 & 2,58 & 5 & - & + \\
\hline 5 & 1,58 & 5 & - & + \\
\hline 6 & 308 & 0,5 & - & + \\
\hline 7 & & & + & \\
\hline 8 & 158 & 1,5 & - & + \\
\hline \multirow[t]{3}{*}{9} & 0,758 & 2 & & - \\
\hline & 1,58 & 2 & - & + \\
\hline & 3.08 & 2 & & + \\
\hline 10 & 1,58 & 2 & - & + \\
\hline \multirow[t]{2}{*}{11} & 1,58 & 1,5 & - & - \\
\hline & & 3 & & - \\
\hline 12 & & & + & \\
\hline
\end{tabular}

+ left heart contrast obtained

-: no left heart contrast seen

wedge position as used for the dextrose injections attempting to achieve LHEC.

\section{RESULTS}

Method 1: physical cavitation-ether

Two of the 10 pigs had LHEC after initial right heart saline injections, and were excluded from further analysis due to the presumed presence of cardiac or pulmonary shunts. Six of the remaining eight animals had LHEC after ether injections (Fig. 1). Since these volumes of intravascular ether are frequently toxic in pigs, we always saw hypotension and occasionally asystole in the pigs after 2 or $3 \mathrm{~cm}^{3}$ of ether.

\section{Method 2: chemical reaction-hydrogen peroxide}

Two of the 12 pigs had initial LHEC after right heart saline injections, and were excluded from further analysis due to presumed cardiac or pulmonary shunting.
Eight of the remaining 10 pigs had LHEC after hydrogen peroxide injections (Fig. 2).

In one pig (number 9) a threshold effect for LHEC could be demonstrated, such that all injections of $\mathrm{H}_{2} \mathrm{O}_{2}$ with a concentration at or above $1.5 \%$ yielded LHEC and those below did not. This was repeatable for 8 injections crossing the threshold concentration 4 times.

Method 3 (animal studies): autopsies of rabbits subjected to pulmonary wedge injections

One rabbit died during the night between the experimental protocol and the day of scheduled sacrifice. At its autopsy, edematous lungs, renal tubular necrosis, and a large myocardial infarction were found. The other 6 rabbits survived to sacrifice and were apparently healthy at the time of sacrifice, $24 \mathrm{hr}$ after the experimental protocol. Three of these 6 rabbits had small myocardial infarctions at autopsy. None of them had renal 


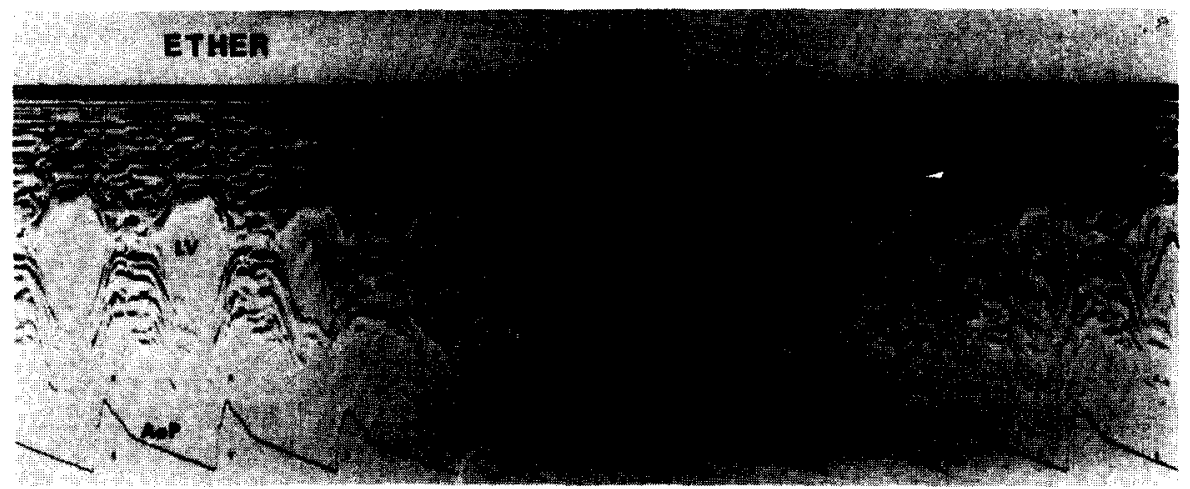

Fig. 1. $M$-mode echocardiogram recorded from an anesthetized pig with the transducer sutured directly onto the left ventricular free wall epicardium. An injection of ether into the right heart causes contrast (arrows) in the left ventricle (LV). AoP: aortic pressure.

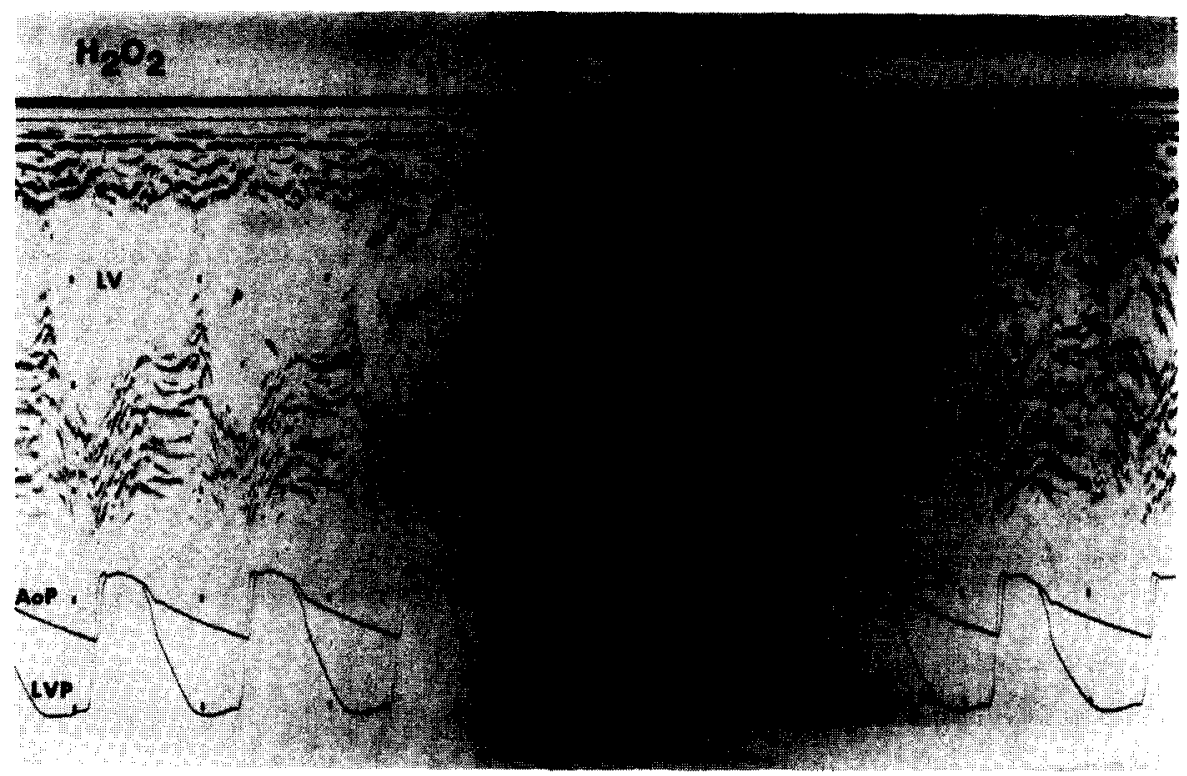

Fig. 2. $M$-mode echocardiogram recorded from an anesthetized pig with the transducer sutured directly onto the left ventircular free wall epicardium. An injection of hydrogen peroxide into the right heart causes contrast (arrows) in the left ventricle (LV). AoP: aortic pressure, LVP: left ventricular pressure. 


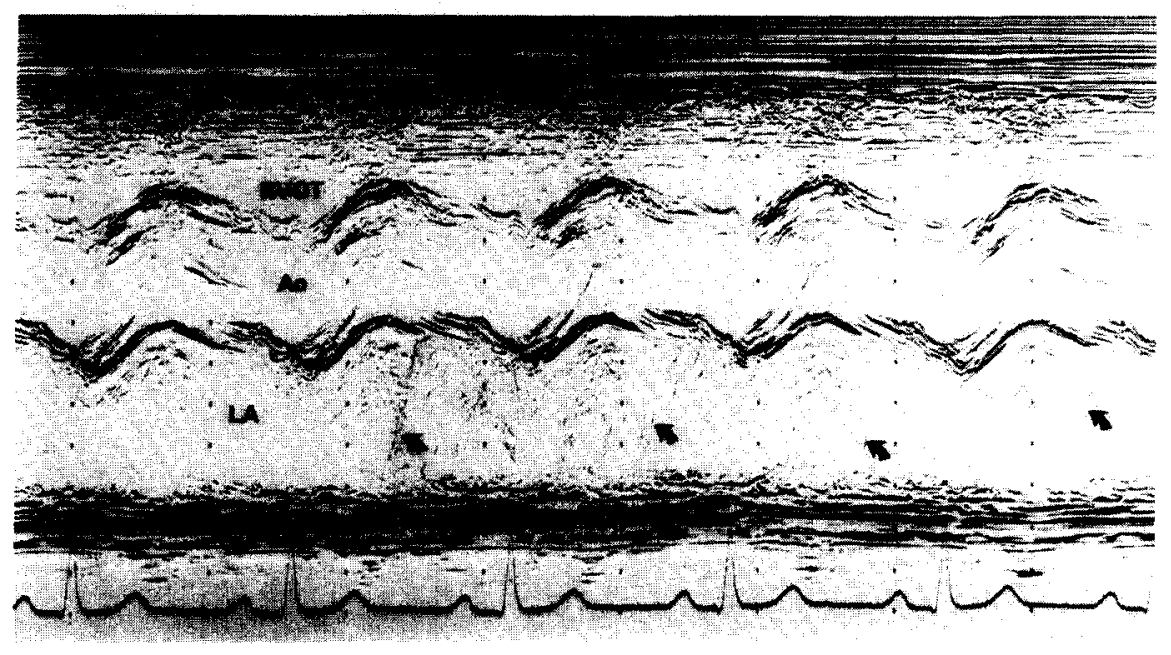

Fig. 3. $M$-mode echocardiogram in a human subject after injection of $10 \mathrm{~cm}^{3}$ of $5 \%$ dextrose solution through a catheter in the pulmonary wedge position. Note the appearance of fine contrast in the left atrium (arrows). Ao: aorta, LA: left atrium, RVOT: right ventricular outflow tract. Reproduced with permission from the British Heart Journal.

a)

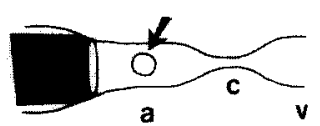

c)

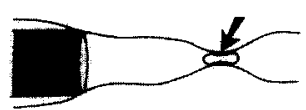

b)

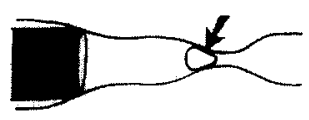

d)

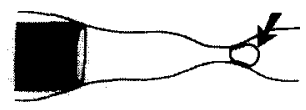

Fig. 4. Diagrammatic representation of proposed mechanism by which microbubbles of gas larger than the pulmonary capillary diameter may pass through the lungs to yield LHEC. (a) A bubble of gas (arrow) injected through a catheter (shaded) in the wedge position enters a pulmonary arteriole (a). (b) Due to the hydrodynamic driving force applied to the local pulmonary circulation due to an injection directly in the wedge position, the bubble is deformed and forced into a pulmonary capillary (c). (c) The bubble elongates and may even fill a capillary entirely with air. (d) The bubble emerges on the venous side (v) of the pulmonary capillary bed. 
lesions or pulmonary injury that could be attributed to the wedge injections.

\section{Method 3 (human): pulmonary wedge in- jections}

No subject has symptoms or worsening of cardiopulmonary status in relation to wedge injections. Eleven of the 15 patients with wedge injections through Cournand catheters achieved LHEC, whereas only 1 of 8 subjects with injections through Swan-Ganz catheters achieved LHEC. An example of a positive study is shown in Fig. 3. LHEC was only attained in the patients where no retrograde flow of X-ray contrast agent around the catheter was noted during wedge injection of the nonionic radiographic contrast agent.

\section{DISCUSSION}

As predicted from the physiologic understanding of microbubbles described earlier, both ether and hydrogen peroxide can pass the pulmonary capillaries in liquid from and yield a gas phase, thus ultrasonic contrast, on the left side of the heart. Though small amounts of each of these substances can be administered in humans, we feel that each is probably too toxic to administer safely intravenously in amounts necessary to obtain LHEC. However, a group from Wuhan, China, has reported the use of IV hydrogen peroxide in animals and humans (Wang et al., 1979a, 1979b). They claim acceptable toxiicity. They also note that LHEC was not seen in the absence of intracardiac shunts.

Our studies do not exclude opening of intrapulmonary arteriovenous shunts as a mechanism for transpulmonary transmission of contrast. However, we are unaware of animal studies suggesting that ether, peroxide or wedge injections open such anatomic channels.

Potential toxicity of transpulmonary transmission of ultrasonic contrast is of concern in the light of our autopsy findings in rabbits after wedge injections. We think that the unexpected and serious toxicity (1 large fatal infarction and three small myocardial infarctions out of seven rabbits) relates to our technique of not carefully excluding small amounts of air from the syringe and 3-way stopcock. Due to this tolerance of small amounts of air, the amount of injected air in relation to body weight was many times that used in human contrast studies, either in routine peripheral injections or our wedge in- jection protocol. We assume that the high prevalence of rabbit myocardial infarctions was due to coronary air embolism. Two mechanisms may explain this phenomenon. First it is possible that most rabbits had significant pulmonary arteriovenous shunts. Though pigs and dogs under anesthesia have a prevalence of these shunts that is about $10 \%$ (unpublished observations), we doubt that this occured in most rabbits. The second possibility is diagrammed in Fig. 4. Whereas normally large bubbles of air are retained by the "sieve" action of the pulmonary capillaries (Meltzer et al., 1980a), the increased driving hydrodynamic pressure applied to the capillary bed during a wedge injection may allow deformation of a bubble into a "dumbbell" shape and intact passage through the capillary bed. The hypothesis that high proximal pressures are needed to obtain LHEC after wedge injections is supported by the observation that in humans LHEC only occurs when the catheter is wedged firmly enough to prevent "blowout" and retrograde $X$-ray contrast flow during the wedge injections.

Though this study suggests caution in designing a clinical method for noninvasive LHEC creation, the long experience with ultrasonic contrast injected into the left heart with catheters implies that the presence of microbubbles in sufficient concentration to cause LHEC need not cause an unacceptable toxicity. Nontoxic, noninvasive LHEC creation remains thus a valid research goal and we are optimistic that a better understanding of microbubble physiology-the factors affecting bubble growth and decay-will permit successful attainment of this goal.

Acknowledgement-The authors wish to thank Rob van Bremen and Marita Rutteman for technical assistance, Inge van Balen for aid in manuscript preparation, and Allan Chesters (Dept. of Aero- and Hydrodynamics, Delft University of Technology, Delft, The Netherlands) for manuscript review and many informative discussions about microbubbles.

\section{REFERENCES}

Barrera, J. G., Fulkerson, P. K., Rittgers, S. E. and Nerem, R. M. (1978) The nature of contrast echocardiographic "targets." Circulation 58, Suppl. II, 233 (abstract).

Bommer, W., Neef, J., Neumann, A., Weinert, L., Lee, G., Mason, D. T. and DeMaria, A. N. (1978) Indicator-dilution curves obtained by photometric analysis of two-dimensional echo contrast studies (Abstract). Am. J. Cardiol. 41, 370.

Bommer W. J., Tickner, E. G., Rasor, J., Grehl, T., 
Mason, D. T., DeMaria, A. N. (1980). Development of a new echocardiographic contrast agent capable of pulmonary transmission and left heart opacification following peripheral venous injection. Circulation 62, Suppl. III, III-34.

Enge, I., Nitter-Hauge, S., Andrew, E. and Levorstad, K. (1977) Amipaque: a new contrast medium in coronary angiography. Radiology 125, 317.

Feigenbaum, H., Stone, J. M., Lee, D. A., Nasser, W. K. and Chang, S. (1970) Identification of ultrasound echoes from the left ventricle by use of intracardiac injections of indocyanine green. Circulation 41, 615.

Gramiak, R. and Shah, P. M. (1968) Echocardiography of the aortic root. Invest. Radiol. 3, 356.

Gramiak, R., Shah, P. M. and Kramer, D. H. (1969) Ultrasound cardiography. Contrast studies in anatomy and function. Radiology 92, 939.

Higashiizumi, T., Tashiro, H., Sakamoto, K. and Kanai, H. (1979) The new ultrasonic method for the elimination of the microairbubbles in the extracorporeal circulation. Proc. 2nd Meeting of the World Federation of Ultrasound in Med. \& Biol., p. 372.

Kerber, R. E., Kioschos, J. M. and Lauer, R. M. (1974) Use of an ultrasonic contrast method in the diagnosis of valvular regurgitation and intracardiac shunts. $A m$. J. Cardiol. 34, 722.

Meltzer, R. S., Tickner, E. G. and Popp, R. L. (1980a) Why do the lungs clear ultrasonic contrast? Ultrasound Med. Biol. 6, 263.

Meltzer, R. S., Tickner, E. G., Sahines, T. P. and Popp.
R. L. (1980b) The source of ultrasound contrast effect. J. Clin. Ultrasound 8, 121.

Meltzer, R. S., Serruys, P. W., McGhie, J., Verbaan, N. and Roelandt, J. (1980c) Pulmonary wedge injections may yield left sided echocardiographic contrast. Brit. Heart J. 44, 390.

Plessett, M. S. and Prosperetti, A. (1977) Bubble dynamics and cavitation. Ann. Rev. Fluid Mech. 9, 145. Preis, L. K., Hess, J. P., Austin, J. L., Craddock, G. B., McGuire, L. B. and Martin, R. P. (1980) Left ventricular microcavitations in patients with Beall valves (Abstract). Am. J. Cardiol. 45, 402.

Reale, A., Pizzuto, F., Gioffrè, P. A., Nigri, A., Romeo, F., Martuscelli, E., Mangieri, E. and Scibilia, G. (1980) Contrast echocardiography: transmission of echoes to the left heart across the pulmonary vascular bed. Europ. Heart J. 1, 201.

Sahn, D. J., Williams, D. E., Shackelton, S. and Friedman, W. F. (1974) The validity of structure identification for cross-sectional echocardiography. $J$. Clin. Ultrasound 2, 201.

Schuchman, H., Feigenbaum, H., Dillon, J. C. and Chang, S. (1975) Intracavitary echoes in patients with mitral prosthetic valves. J. Clin. Ultrasound 3, 107.

Wang, X., Wang, J., Huang, Y. and Cai, C. (1979) Contrast echocardiography with hydrogenperoxide, experimental study. Chin. Med.J. 92(9), 595.

Wang, X., Wang, J, Chen, H. and Lu, C. (1979) Contrast echocardiography with hydrogenperoxide, clinical application. Chin. Med. J. 92(10), 693. 